\title{
Helicobacter cinaedi
}

National Cancer Institute

\section{Source}

National Cancer Institute. Helicobacter cinaedi. NCI Thesaurus. Code C86437.

A species of microaerophilic, Gram negative, helical rod shaped bacteria assigned to the phylum Proteobacteria. This species is oxidase and catalase positive, motile by a single polar flagellum and does not hydrolyze hippurate or indoxyl acetate effectively. H. cinaedi is found in the normal gut flora of hamsters and is an opportunistic pathogen in immunocompromised patients causing fever, gastroenteritis, bacteremia and recurrent cellulitis. 